\title{
MODELACIÓN DE LAS DINÁMICAS, ESTIMACIÓN Y ANÁLISIS DE LAS EXPORTACIONES MINERAS: CASO PERUANO
}

\author{
MODELING OF DYNAMICS, ESTIMATION AND ANALYSIS OF MINING \\ EXPORTS: PERUVIAN CASE
}

MARÍA RAQUEL CHAFLOQUE-CÉSPEDES', VÍCTOR MANUEL CHUNG ALVA², ANA BERTHA COTRINA CAMACHO

1 Universidad de San Martín de Porres. mchafloquec@usmp.pe

2 Universidad de San Martin de Porres. vchunga@usmp.pe

3 Universidad de San Martin de Porres. acotrinac@usmp.pe

RESUMEN

Las exportaciones mineras en el Perú son un factor importante en la dinámica de la economía peruana, interviniendo en un $64.9 \%$ del total de las exportaciones. Un elemento importante para los inversionistas en el sector minero, es saber a corto, mediano y largo plazo, el comportamiento de las exportaciones mineras. En este contexto el presente estudio presenta el modelado de las dinámicas, la estimación y el análisis del comportamiento de las exportaciones mineras desde el año 1985 hasta el año 2017. El objetivo principal de esta investigación fue aplicar las metodologías de predicción y suavizamiento para pronosticar a corto plazo las exportaciones mineras. Se utilizó el análisis estadístico descriptivo y la comparación de los métodos predictivos ARIMA y SARIMA. Los resultados presentados pueden ser utilizados por instituciones privadas y/o públicas para tomar decisiones relacionadas a las inversiones en el sector minero. Finalmente, la presente investigación concluye en que el modelo SARIMA es el modelo óptimo para predecir el futuro de las exportaciones mineras.

PALABRAS CLAVE: exportaciones mineras, series de tiempo, ARIMA, SARIMA.

\begin{abstract}
Mining exports in Peru are an important factor in the dynamics of the Peruvian economy, intervening in $64.9 \%$ of total exports. An important element for investors in the mining sector is to know in the short, medium and long term, the behavior of mining exports. In this context, the present study presents the modeling of dynamics, estimation and analysis of the behavior of mining exports from 1985 to 2017. The main objective of this research was to apply predictive methodologies and smoothing to forecast short-term mining exports. Descriptive statistical analysis and comparison of the ARIMA and SARIMA predictive methods were used. The results presented can be used by private and / or public institutions to make decisions related to investments in the mining sector. Finally, the present investigation concludes that the SARIMA model is the optimal model to predict the future of mining exports.
\end{abstract}

KEYWORDS: mining exports, time series, ARIMA, SARIMA. 
INTRODUCCIÓN

El Banco Mundial señalo que en el 2017 las exportaciones a nivel mundial corresponden al $28.52 \%$ del PBI mundial (Banco Mundial, 2017). Asimismo, en el mismo año, según el Indicador de las Perspectivas del Comercio Mundial de la Organización Mundial de Comercio (WTOI por sus siglas en inglés), el comercio internacional a nivel mundial registró un crecimiento de $2.6 \%$ frente al 1.3\% registrado en el 2016. El WTOI ha registrado índices que reportan un crecimiento constante del volumen del comercio de mercancías (WTO, 2017; WTO, 2018).

La WTOI ha reportado que los principales países exportadores a nivel mundial son los países de China, la Unión Europea, India, Estados Unidos y Turquía. Asimismo, se reportó que las economías en desarrollo han aportado en el 42\% de comercio exterior en el 2016. Las economías latinoamericanas en desarrollo aportan el 5.7\%; destacando Argentina, Perú, República Dominicana, Costa Rica y Paraguay (WTO, 2017).

Las exportaciones peruanas en el año 2016, aportaron el $22.1 \%$ del PBI. Las exportaciones peruanas están compuestas por exportaciones de productos tradicionales (pesqueros, agrícolas, mineros, petróleo y gas natural) y no tradicionales (agropecuarios, pesqueros, textiles, maderas y papeles, químicos, minerales no metálicos, siderometalúrgicos y joyería, metal-mecánicos y otros). En el Perú, las exportaciones mineras aportan con más del 60\% del total de exportaciones en términos de valor FOB, mientras que en términos de volumen las exportaciones mineras compensan las caídas de otros productos. Los productos considerados dentro de las exportaciones mineras con mayor participación son el oro, cobre y zinc (BCRP, 2016a; BCRP, 2016b; Valdés, 2016).

Las investigaciones recientes sobre la economía peruana explican que el Perú es un país dependiente del sector minero, principalmente las exportaciones mineras, sin embargo, estas pueden verse afectadas por factores económicos, políticos o sociales (Nolazco, et al. 2016; Glave, 2007). Los estudios explican que el sector minero ha influido en la economía peruana probablemente desde el año 1593, y ha sido uno de los principales sectores que durante los ciclos económicos ha influido en el período de recuperación (Seminario, 2016).

El sector minero como actividad primaria toma la mayor importancia con respecto a la participación del PBI peruano, en su aporte económico a nivel regional y local a la com- plementación de este sector para alcanzar productividad no solo al sector minero, también genera externalidades en otros sectores como transporte, logística y manufactura. En consecuencia, generando empleo directo e indirecto y contribuyendo con la inversión privada en el Perú, generando un efecto multiplicador, incrementando la demanda interna al considerar la exportación minera peruana.

La evolución macroeconómica del Perú en los últimos 16 años ha sido favorable para el Perú. Una de las contribuciones importantes ha sido por parte del sector minero en la Bolsa de Valores de Lima (BVL). La recuperación de la BVL entre los años 2016 y 2017. En cuanto al monto regular negociado este se incrementó a $52 \%$ en 2017. Este comportamiento responde al escenario de corto plazo y largo plazo: vinculado al crecimiento de la economía de China, en la actualidad muestra signos de recuperación de los precios de los metales como el cobre y zinc entre otros (Tramontana, 2018).

Econométricamente, se han desarrollado investigaciones para describir y analizar las series de las exportaciones en diferentes sectores. Hasta el momento la academia ha realizado investigaciones que utilizan métodos de predicción en las exportaciones. Lanteri (2010), aplicó modelos de VAR Bayesianos y Factor Aumentado VAR (FAVAR) para pronosticar las exportaciones argentinas. Benavente (2017), utilizaron diferentes métodos predictivos (promedio, promedio móvil de dos periodos, suavización exponencial y tendencia), para analizar series de tiempo trimestrales en los valores FOB de las exportaciones de atún, los autores concluyeron que la mejor técnica para analizar estos datos es la de promedios. Otro método utilizado para predecir las exportaciones es el ARIMA, Aguilar y Valdivia (2011) utilizaron este método para predecir los precios de exportación de gas natural en Bolivia. En la misma línea de investigación Alvarado et al. (2014), analizaron las exportaciones del sector petrolero en Ecuador y pronosticaron los precios del sector mediante el modelo ARIMA, durante el periodo de la crisis financiera mundial. Al-Zyoud y Elloumi (2017), analizaron las tendencias de las exportaciones e importaciones de Canadá (1981 - 2014) utilizando la regresión log-lineal junto con un modelo de media móvil integrada autorregresiva (ARIMA).

A pesar de que la variable analizada ya ha sido desarrollada y analizada por entidades públicas, así como también por la academia, la 
presente investigación analiza las exportaciones de uno de los productos más importantes en la economía peruana, ya que este tiene mucha influencia en el comportamiento de la balanza comercial y el Producto Bruto Interno (PBI). Por tal motivo es necesario disponer de adecuados análisis de esta variable ya que resulta útil a fin de diseñar políticas que eviten las crisis ocurridas en el pasado.

El propósito de este trabajo es aplicar metodologías de predicción y suavizamiento para pronosticar a corto plazo las exportaciones mineras. Por tal motivo, en este estudio demostraremos como podemos predecir el futuro respaldando nuestra predicción en datos pasados.

\section{MATERIALES Y MÉTODOS}

El presente estudio ha considerado datos de series de tiempo para analizar la tendencia a largo plazo y como esta se relaciona con el contexto político vivido en el periodo estudiado. Los datos mensuales sobre la exportación minera del periodo 1985 - 2017, se han extraído de la base de estadísticas económicas del Banco Central de Reservas del Perú (BCRP). Los datos sobre las exportaciones mineras están disponibles en valor FOB, en millones de dólares americanos. La base de datos está disponible en el siguiente link: https://estadisticas.bcrp.gob.pe/estadisticas/series/mensuales/resultados/PN01475BM/ html.

Se realizó un análisis estadístico descriptivo de la serie de tiempo, así como también se graficaron los datos para observar el comportamiento de la serie en función del tiempo con el fin de comprobar si la media y la varianza del proceso permanecen constantes. Para la elección de los modelos descritos anteriormente se utilizó la metodología Box - Jenkins, la cual tiene 3 pasos:

- Identificación y selección del modelo (Estacionariedad, estacionalidad y de medias móviles).

- Estimación de los coeficientes que mejor se ajustan a los parámetros escogidos por medio de algoritmos computacionales.

- Validación del modelo obtenido.

Para realizar el análisis estadístico exploratorio y la técnica de predicción se utilizó el programa Eviews y el programa R studio 3.2.5. Se utilizaron los paquetes forecast, TTR, ggplot2 y se utilizaron los comandos presentados en el anexo 1. Para el objeto central de este análisis se llevó a cabo un proceso metodológico que consiste en un análisis preliminar para la determinación de la tendencia de la serie de tiempo anual. Se diagnosticó considerando la función de autocorrelación (ACF) y la función de autocorrelación parcial (PACF). Para la selección del modelo se consideró los criterios de información de Akaike (AIC) y el RMSE. Cabe resaltar que en la presente investigación se busca un enfoque de aproximación a una realidad la cual es reconocida como compleja y por tanto no se espera un ajuste exacto.

MODELOS DE PRONÓSTICO

Según López \& Martínez (2013), explican que las series de tiempo se reducen por sí solas a una simple organización temporal de muestras que describen parámetros básicos de un proceso estocástico, y estas son modeladas por los siguientes modelos:

MODELO AUTORREGRESIVO (AR)

El modelo se describe en la ecuación (1), el valor actual de la serie se expresa como una función de los valores que tomo la misma en las p muestras anteriores ponderados por un factor y de una perturbación aleatoria presente.

$$
\phi_{p}(L) Y_{t}=\mu_{t}
$$

\section{MODELO DE MEDIAS MÓVILES (MA)}

El valor de la serie estacionaria se desplaza alrededor de un valor medio El valor de la serie estacionaria se desplaza alrededor de un valor medio El valor de la serie estacionaria se despla$z a$ alrededor de un valor medio $\mu$ en el tiempo presente $t$ es ocasionado por infinitas perturbaciones ocurridas en el pasado, ponderadas por un factor que mide la influencia de cada una de ellas en el valor presente de la serie (ver ecuación 2).

$$
Y_{t}-\mu=\Theta_{q}(L) u_{t}
$$

MODELO ARMA

En la ecuación 3, este modelo representa una serie de tiempo como una combinación de los dos modelos anteriores:

$$
\phi_{p}(L) Y_{t}=\Theta_{q}(L) u_{t}
$$




\section{MODELO ARIMA (AUTOREGRESSIVE INTEGRATED} MOVING AVERAGE)

El modelo ARIMA destaca por ser simple y práctico para modelar y pronosticar las series de tiempo. El modelo ARIMA es considerado por muchos académicos para realizar pronósticos o predicciones en series de tiempo macroeconómicas, debido a que considera registros de datos con tendencia (Amaris et al. 2017).

El método consiste en la combinación de un término autorregresivo (AR) y un término de promedio móvil (MA) con un elemento diferenciador dado por la letra I basado en un estudio realizado por Yaglom (1955). En general estos modelos se referencian con la palabra ARIMA (p, d, q). Donde (p) se refiere al orden del modelo autorregresivo; (d), al término de diferenciación, y (q), al término de media móvil con q términos de error (ver ecuación 4).

$$
\phi_{p}(L)(1-L)^{d}\left(Y_{t}-\mu\right)=\Theta_{q}(L) u_{t}
$$

\section{MODELO SARIMA (SEASONAL AUTOREGRESSIVE INTEGRATED MOVING AVERAGE)}

Es autorregresivo e integrado de promedio móvil estacional, se basa en ARIMA, con algunos de sus coeficientes en cero y componentes adicionales para integrar el comportamiento estacional de la serie (ver ecuación 5).

$$
\phi_{P}(L) \phi_{p}\left(1-L^{S}\right)^{D}(1-L)^{d}\left(Y_{t}-\mu\right)=\Theta_{Q}(L) \Theta_{q}(L) u_{t}
$$

RESULTADOS

ANÁLISIS ESTADÍSTICO DESCRIPTIVO DE LA VARIABLE EXPORTACIÓN DE PRODUCTOS MINEROS

Los valores FOB de las exportaciones de productos tradicionales mineros, fluctuaron entre 65.8 millones y 2778.72 millones de dólares americanos. El valor mínimo de las exportaciones se dio exactamente en febrero de 1986 durante el primer gobierno de Alan García, mientras que el valor máximo de las exportaciones mineras se dio exactamente en agosto del 2011 durante el gobierno de Ollanta Humala.

El promedio de las exportaciones desde enero de 1985 a abril del 2017 es 768.35 millones de dólares americanos. En el gráfico de dispersión se observa que las exportaciones tienen tendencia y se observa una clara pendiente positiva. Sin embargo, también se observan variaciones irregulares casi por los meses de los años 2008 y 2009 (ver Figura 1).

Asimismo, en la Figura 1, se muestra los valores FOB de las exportaciones durante seis gobiernos. Los primeros datos pertenecen al Primer gobierno de Alan García Pérez (1985 1990), como se observa en la gráfica, durante este gobierno los datos registrados de exportación se mantienen constantes a pesar de las políticas exteriores aplicadas en dicho gobierno, los productos tradicionales se mantuvieron en recesión.

El panorama de exportaciones mineras en el Primer y segundo gobierno de Alberto Fujimori (1990 - 2000), los datos registrados se mantienen constantes sin presentar una pequeña tendencia creciente, no existieron muchas variaciones en las exportaciones y eso se debe a que las políticas aplicadas no eran totalmente de una economía abierta.

El crecimiento de los valores FOB de las exportaciones inicia con las políticas aplicadas en el gobierno de Alejandro Toledo (2001 - 2006), aplicando políticas de economía abierta y basando el crecimiento de la economía en las exportaciones de productos tradicionales y no tradicionales.

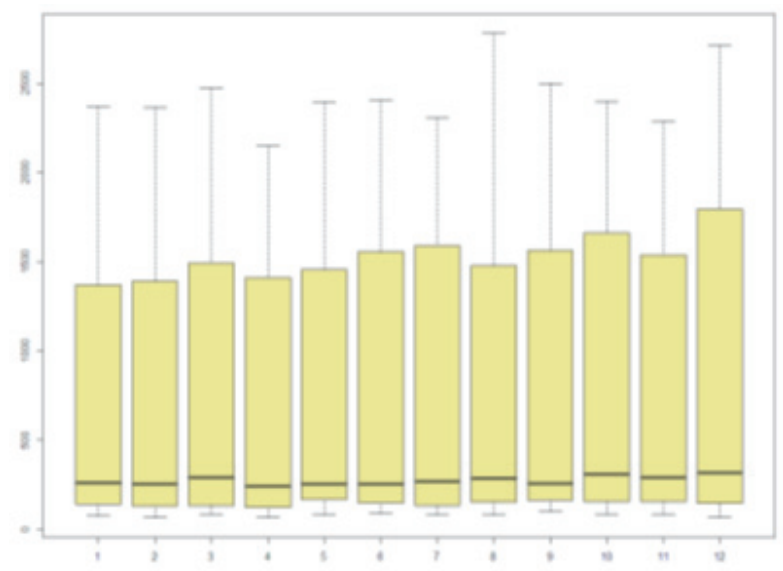

Figura 1. Gráfico de dispersión de exportaciones mineras - valor FOB mensual (1985 - 2017)

En la Figura 2, el gráfico de dispersión se refleja al momento de analizar de manera descriptiva por medio del diagrama de cajas y el histograma. En el diagrama de cajas, se observa el desplazamiento a la izquierda en los 12 meses de la serie de tiempo, todos los meses presenta menor variabilidad al 50\%. En la Figura 3, el histograma presenta un comportamiento asimétrico, esto no ayuda mucho a la descripción de la serie de tiempo. 


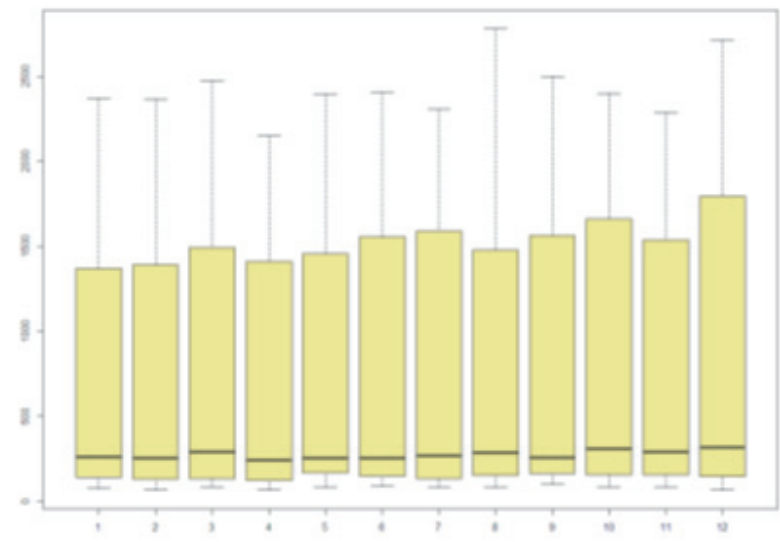

Figura 2. Diagrama de cajas de exportaciones mineras - valor FOB mensual (1985 -2017).

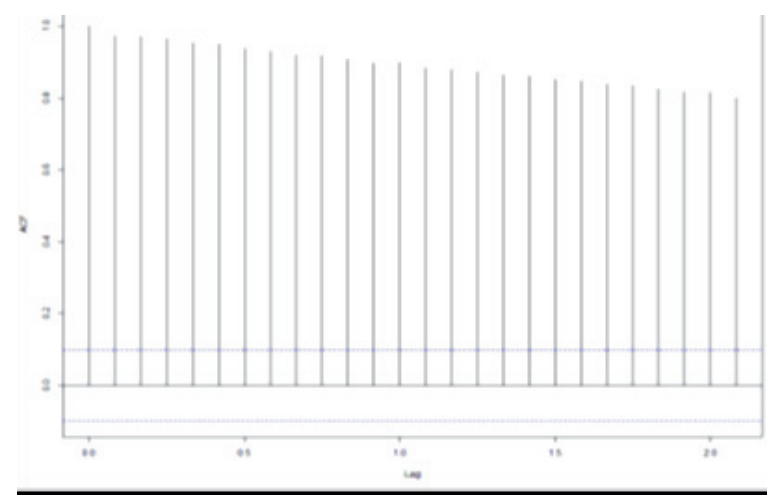

Figura 3. Histograma de exportaciones mineras - valor FOB mensual (1985 -2017).

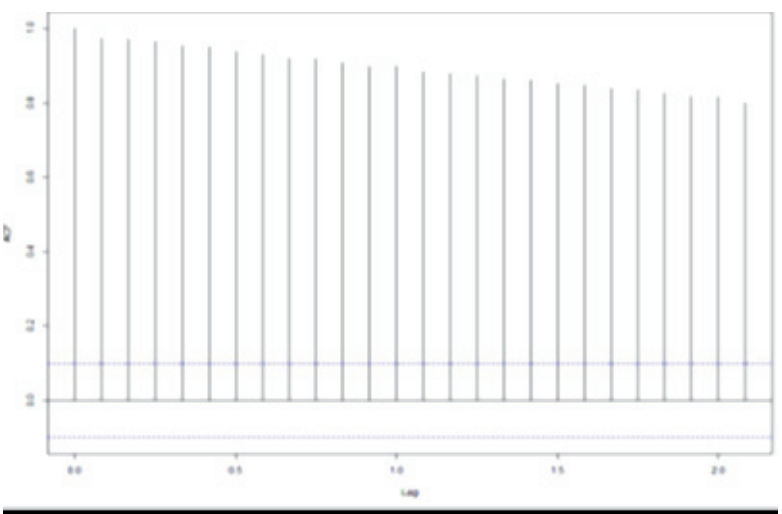

Figura 4. Gráfico de normalidad de exportaciones mineras - valor FOB mensual (1985-2017)

Con respecto a la prueba de normalidad, tal como se observa en el Figura 4 y con la prueba estadística de Shapiro-Wilk ( $\mathrm{W}=0.79761$, $\mathrm{p}$-value $=2.2 \mathrm{e}-16$ ), existe suficiente evidencia estadística para rechazar la hipótesis nula. En efecto, las exportaciones tradicionales mineras presentan distribución asimétrica. Analizando la función de autocorrelación de la serie de tiempo, se observa una clara tendencia, así como también lo confirman los primeros retrasos registrados ( $\mathrm{r} 1=0.973, \mathrm{r} 2=0.972)$ los cuales disminuyen hasta r21 $=0.838$, como se observa en la Figura 5, los coeficientes de las ACF no decaen rápidamente y esto nos da un claro indicio de falta de Estacionariedad en media, lo cual nos lleva a tomar primeras diferencias en la serie original.

En la Figura 6, se observa que la función de autocorrelación parcial (PACF) estimada validan la no estacionalidad de los datos. Por otro lado, el PACF no presenta una estructura de coeficientes significativos para retardos periódicos largos. La PACF muestra dos barras sobresalientes en el primer y segundo desfase con un valor de PACF de 0.472 y 0.148 respectivamente, y tres desfases más durante toda la función.

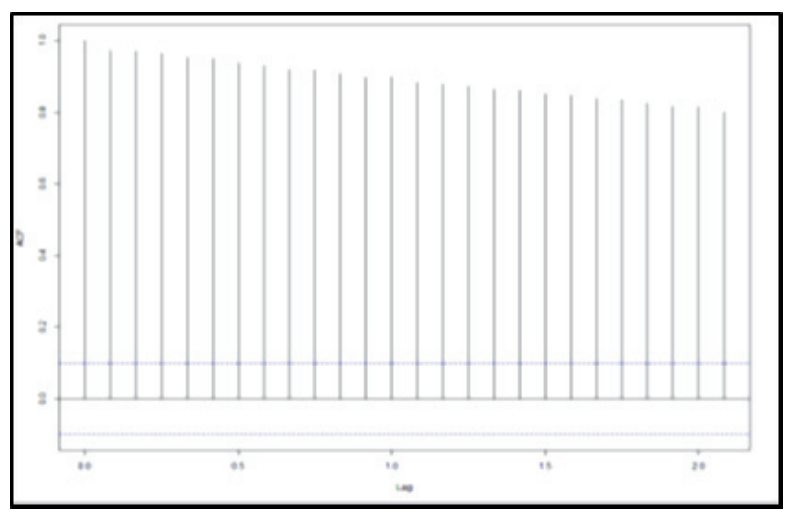

Figura 5. Gráfico función de autocorrelación.

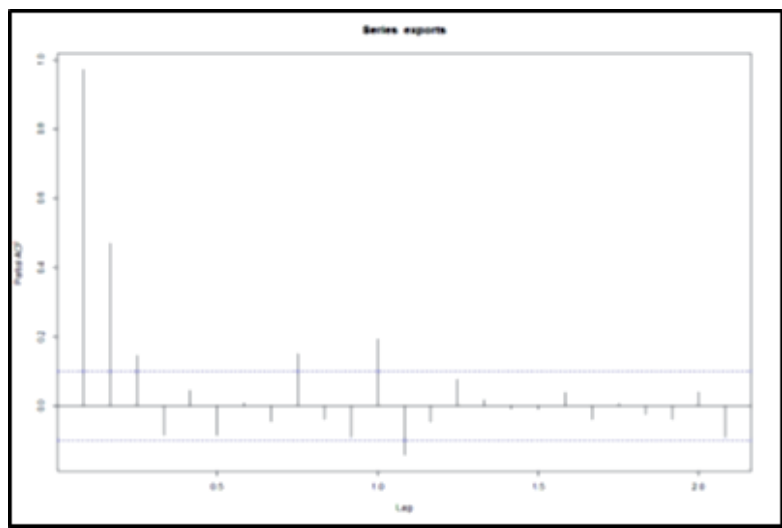

Figura 6. Gráfico función de autocorrelación parcial.

\section{APLICACIÓN DE LOS MODELOS ARIMA Y SARIMA MODELO ARIMA}

- IdentificAción DEl MOdelo ARIMA: La Figura 7, muestra las transformaciones de la serie de tiempo en varianza y en media dado que la serie de tiempo de las exportaciones mineras es no estacionaria por lo cual se transformaron los datos 

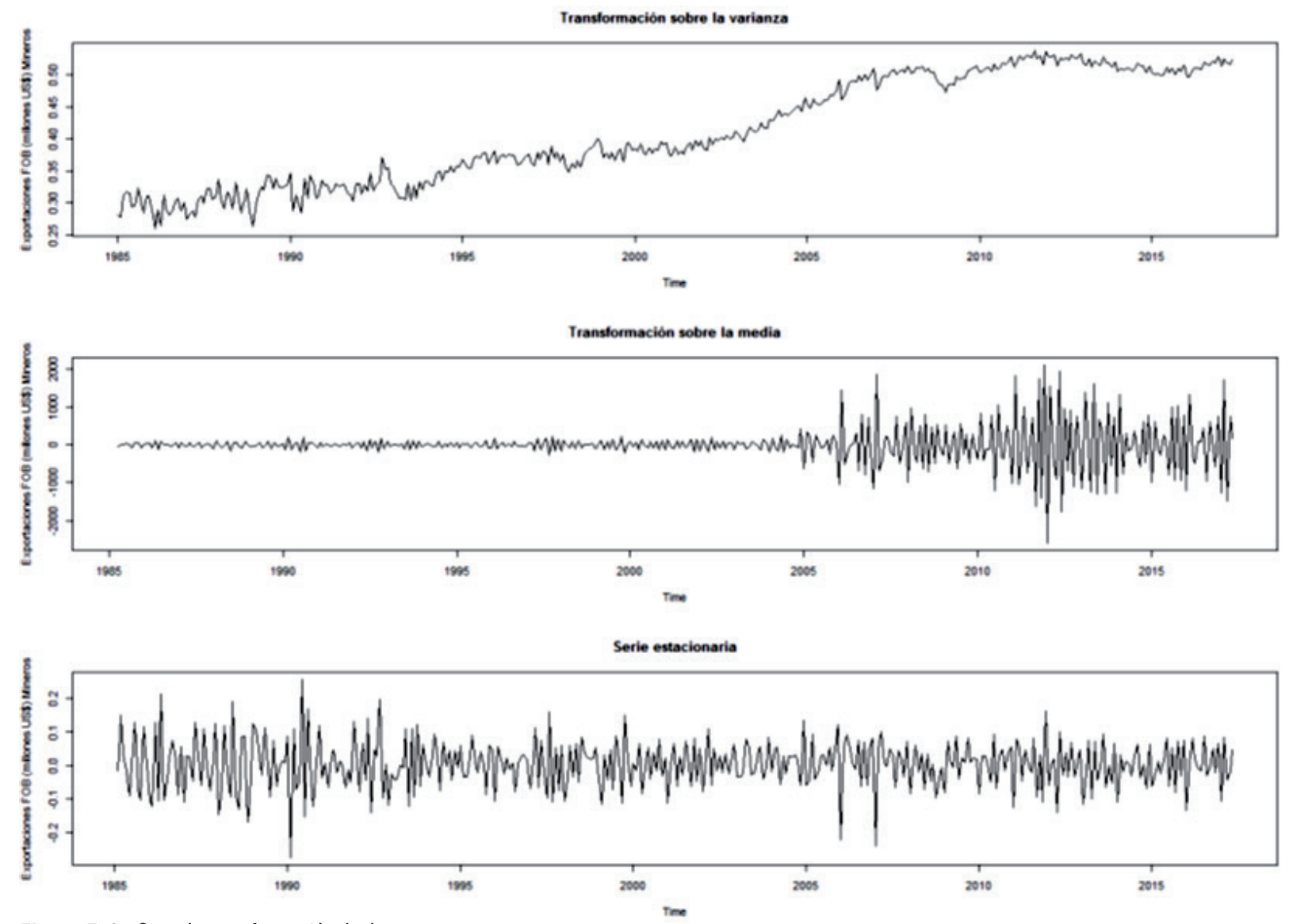

Figura 7. Graficas de transformación de datos.

sobre la varianza, utilizando logaritmo natural, sin embargo, la serie de tiempo se mantuvo siendo no estacionaria, por lo cual se aplicó la transformación en medias para hacer la serie de tendencia (media constante).

La ACF y el PACF corroboran que la serie de tiempo ya ha sido transformada a estacionaria (ver Figura 8). Como se puede observar, estas funciones arrojan valores significativos en los primeros tres retardos y aquellos múltiplos de 12, lo cual muestra el comportamiento periódico de la serie. Además, como se describe en la siguiente sección, a partir de estas graficas se establecerá el orden de los demás parámetros de cada modelo.

- Estimación de parámetros del modelo ARIMA: En la Figura 8, se puede apreciar que también dentro de los periodos no estacionales las tres primeras autocorrelaciones parciales y la primera autocorrelación simple son significativas. Para los periodos estacionales las autocorrelaciones parciales de los retardos 12 y 24 son significativos, y entre las simples sólo la del retardo 12 es significativa.

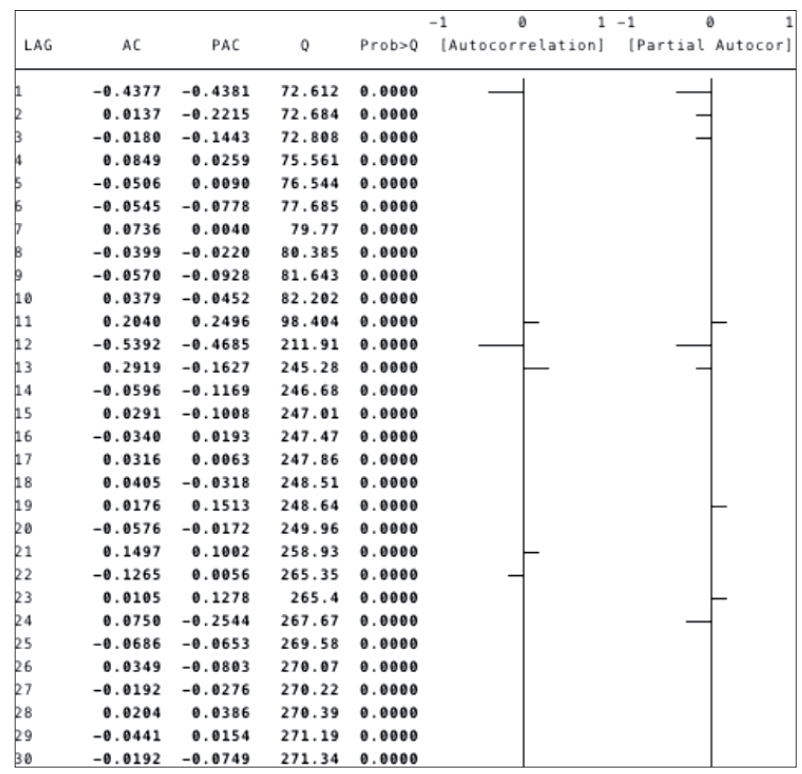

Figura 8. Gráficas del ACF y PACF.

Basado en lo mencionado anteriormente se consideraron todas las combinaciones posibles, tanto con esquema aditivo como en un multiplicativo. En la siguiente tabla se presentan sólo las combinaciones en las cuales los coeficientes fueron significativos: 
TABLA 1. CRITERIO DE PENALIZACIÓN DE AKAIKE Y RSME PARA LOS DIVERSOS MODELOS

\begin{tabular}{llcc}
\hline ESquEMA & MODELO PROPUESTO & AIC & RSME \\
\hline Aditivo & ARIMA $(3,1,0)$ & -176.281 & 0.189 \\
& ARIMA $(0,1,1)$ & -177.648 & 0.190 \\
& ARIMA $(24,1,0)$ & -321.119 & 0.154 \\
& ARIMA $(36,1,0)$ & -336.119 & 0.150 \\
& ARIMA $(3,1,12)$ & $\mathbf{- 4 0 3 . 7 7 4}$ & $\mathbf{0 . 1 3 5}$ \\
Multiplicativo & SARIMA $(3,1,0)(2,1,0)_{12}$ & -341.278 & 0.150 \\
& SARIMA $(3,1,0)(3,1,0)_{12}$ & -358.838 & 0.145 \\
& SARIMA $(0,1,1)(3,1,0)_{12}$ & -360.359 & 0.146 \\
& SARIMA $(0,1,1)(0,1,1)_{12}$ & -405.669 & $\mathbf{0 . 1 3 5}$ \\
\hline
\end{tabular}

Entre los modelos estimados considerando un esquema aditivo se encontró que el ARIMA $(3,1,12) 12$ es el que tiene los menores valores. El modelo puede ser expresado como:

$\left(1+0.5637 L+0.3191 L^{2}+0.3191 L^{3}\right)(1-L) Y_{t}=$ $=\left(1-0.3191 L^{12}\right) e_{t}$

- VALIDACIÓN de Los MODELos: Para evaluar el modelo estimado se presenta el correlograma de los residuales en la Figura 9. Los residuales del modelo estimado tienen el comportamiento de una variable de ruido blanco, es decir un comportamiento estacionario. Esto se confirma utilizando la prueba Portmanteau y la prueba de Bartlett's de ruido blanco (ver Tabla 2).

TABLA 2. PRUEBA DE RUIDO bLANCO PARA LOS RESIDUALES DEL MODELO ARIMA $(3,1,12)$

\begin{tabular}{lcc} 
PRUEBA & VALOR & P \\
\hline Estadístico Portmanteau (Q) & 35.5099 & 0.6725 \\
Estadístico de Bartlett's & 0.4296 & 0.9927 \\
\hline
\end{tabular}

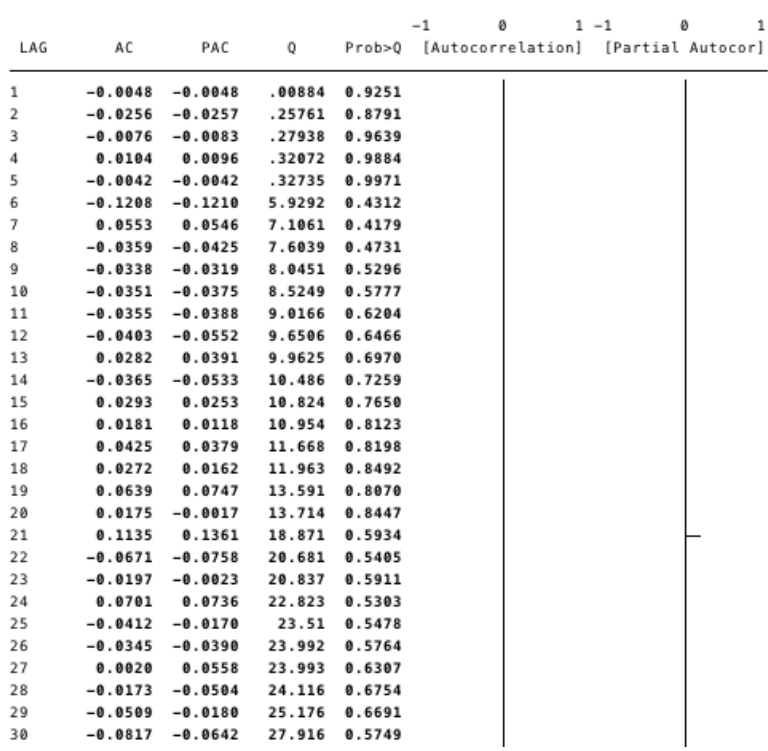

Figura 9. Correlograma de los residuales del modelo ARIMA $(3,1,12)$
- Predicción utilizando ARIMA: Los valores pronosticados se presentan en la figura 10. Con estos pronósticos se obtuvo un valor RMSE igual a 0.135 , que es el menor valor obtenido entre los modelos con esquema aditivo.

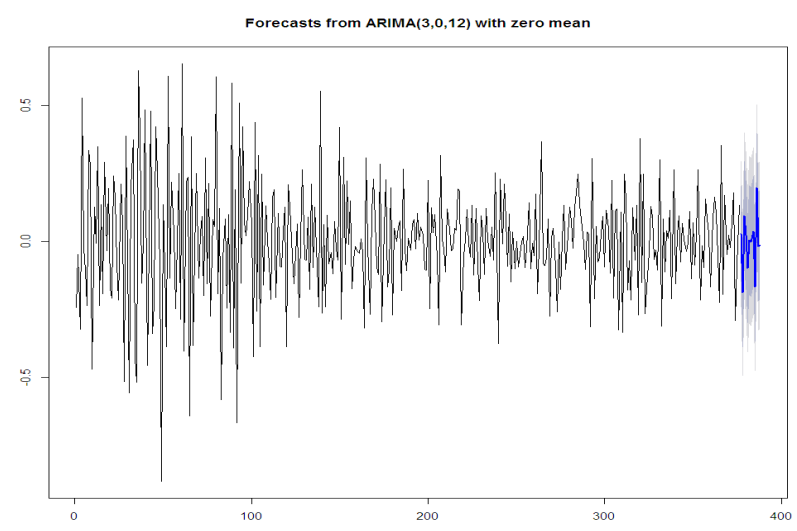

Figura 10. Valores observados y pronóstico de las exportaciones mineras con el modelo ARIMA $(3,1,12)$.

MODELO SARIMA

- IDENTIFICACión DEL MOdelo: Al igual que en el modelo ARIMA nos basaremos en los gráficos de AFC y PAFC, a partir de la Figura 11 se indicó que los periodos estacionales las autocorrelaciones parciales de los retardos 12 y 24 son significativos, y entre las simples sólo la del retardo 12 es estadísticamente significativamente diferente de cero que cae fuera del Intervalo de confianza del 95\%. La observación de PACF muestra la insignificancia de otros valores después de la primera observación.

- Estimación de parámetros del MODELO SARIMA: La Tabla 1 presenta los diversos modelos SARIMA de los cuales el SARIMA $(0,1,1)(0,1,1) 12$ es él que tiene el menor valor AIC, el cual puede ser expresado como:

$(1-L)\left(1-L^{12}\right) Y_{t}=\left(1-0.939 L^{12}\right) e_{t}$

- Validación del modelo SARima: Para realizar la validación, se utilizó el correlograma de los residuales para determinar la aptitud de los datos, la salida de ACF y PACF se presenta en la Figura 11. El resultado muestra que ninguno de los ACF y PACF es individualmente estadísticamente significativo, lo que significa que los correlogramas afirman que los residuos estimados a partir de la regresión son ruido aleatorio puro, por lo tanto, el modelo SARIMA especificado 
es confirmado y afirmado como adecuado. Asimismo, se confirma utilizando la prueba Portmanteau y la prueba de Bartlett's de ruido blanco (ver Tabla 3).

TABLA 3. PRUEBA DE RUIDO BLANCO PARA RESIDUALES DEL
\begin{tabular}{lcc} 
MODELO SARIMA $(\mathbf{0}, 1,1)(\mathbf{0}, \mathbf{1}, \mathbf{1})$ 12 & & \\
PRUEBA & VALOR & P \\
\hline Estadístico Portmanteau (Q) & 38.7868 & 0.5248 \\
Estadístico de Bartlett's & 0.6469 & 0.7967 \\
\hline
\end{tabular}

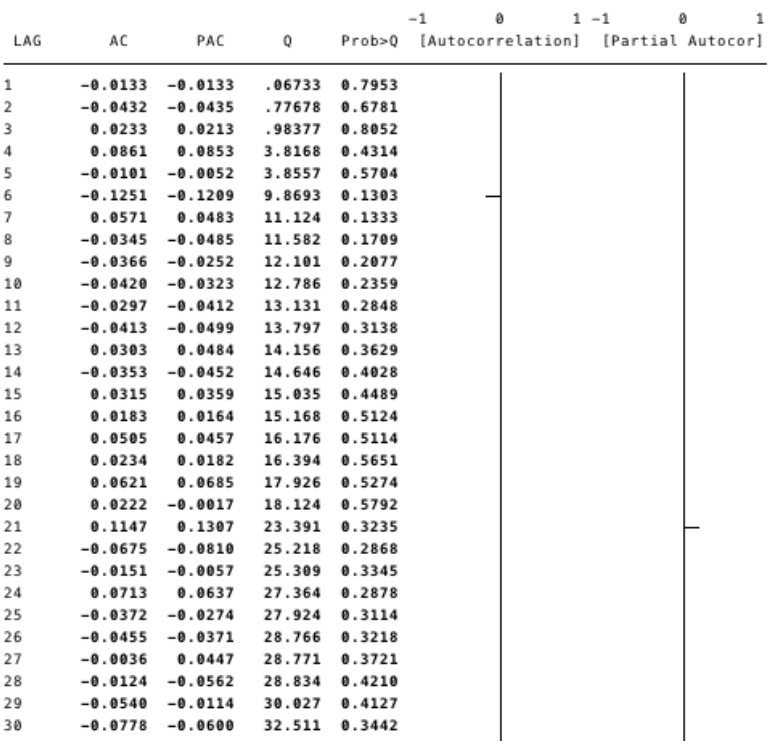

Figura 11. Correlograma de los residuales del modelo SARIMA $(0,1,1)(0$, $1,1)_{12}$.

- Predicción utilizando SARima: En la Figura 12, se observa el pronóstico del modelo presentado en la ecuación 7. Se utilizó el rango de 32 años entre 1985 y 2017. En la Tabla 4, se presentan los valores FOB de las exportaciones mineras con sus respectivos parámetros, considerando un nivel de confianza de 95\%. Con estos pronósticos se obtuvo un valor RMSE igual a 0.135 , que es el menor valor obtenido entre los modelos con esquema multiplicativo.

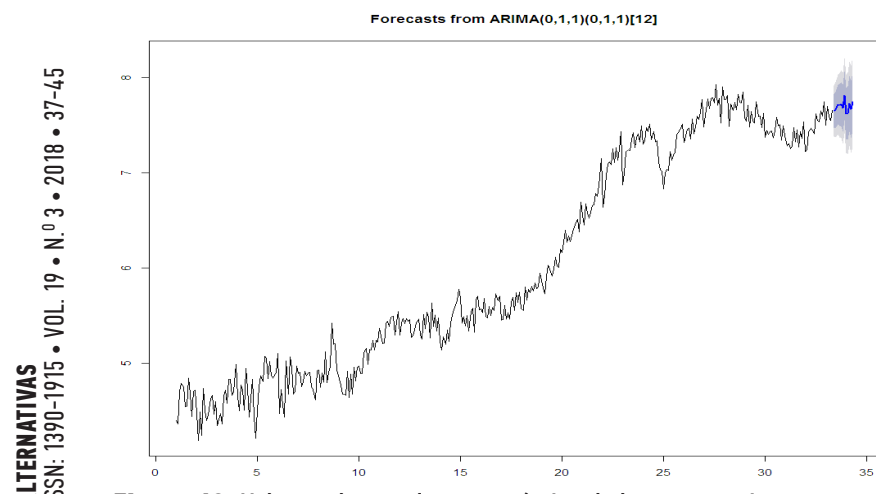

Figura 12. Valores observados y pronóstico de las exportaciones mineras del modelo SARIMA $(0,1,1)(0,1,1)_{12}$
TABLA 4. VALORES PRONOSTICADOS DEL MODELO SARIMA $(0,1,1)$ $(0,1,1)_{12}$

\begin{tabular}{|crcc|}
\hline PERIODO & ESTIMACIÓN & INFERIOR (95\%) & SUPERIOR (95\%) \\
\hline Jun -17 & 2106.118 & 1614.057 & 2748.189 \\
\hline Jul -17 & 2126.239 & 1588.819 & 2845.443 \\
\hline Ago -17 & 2245.664 & 1639.501 & 3075.941 \\
\hline Set -17 & 2235.752 & 1597.318 & 3129.362 \\
\hline Oct - 17 & 2256.095 & 1579.412 & 3222.696 \\
\hline Nov - 17 & 2165.332 & 1486.996 & 3153.110 \\
\hline Dic - 17 & 2467.927 & 1664.072 & 3660.095 \\
\hline Ene - 18 & 2041.991 & 1353.017 & 3081.802 \\
\hline Feb - 18 & 2057.363 & 1340.537 & 3157.497 \\
\hline Mar - 18 & 2267.709 & 1453.946 & 3536.930 \\
\hline Abr - 18 & 2139.143 & 1350.328 & 3388.755 \\
\hline May - 18 & 2317.729 & 1441.194 & 3727.375 \\
\hline & & & CONCLUSIONES \\
\hline
\end{tabular}

Los resultados obtenidos en este estudio muestran que el método de mejor proyección fue el SARIMA $(0,1,1)(0,1,1) 12$. La predicción realizada con el modelo SARIMA nos dice que los valores FOB de las exportaciones tradicionales mineras mantendrán la tendencia de crecimiento, se pronostica que en los próximos 12 meses se tendrá un crecimiento de $9 \%$ aproximadamente, esto surgirá con facilidad, si es que se hace un seguimiento constante del proceso de implementación de políticas monetarias que mantengan el tipo de cambio en los parámetros definidos por el Banco Central de Reservas del Perú.

La estimación a corto plazo de las exportaciones tradicionales mineras, realizado con el modelo SARIMA permite representar de manera óptima el comportamiento de esta y otras variables relacionadas. Los resultados encontrados nos dan una visión de cómo será la evolución de las exportaciones durante el próximo año, sin embargo, el pronóstico no será real si es que no se aplican los esfuerzos rápidos y decisivos de las autoridades pertinentes. En términos de diseño de políticas, documentación y establecimiento de objetivos, el documento de reforma de la política de exportaciones es adecuado.

Pronosticar el comportamiento a corto plazo de las exportaciones tradicionales mineras es importante no solo para el escenario internacional, también es importante para los inversionistas en la Bolsa de Valores de Lima (BVL), ya que la mayoría de acciones que tiene la BVL es del sector minero. Una recomendación para un próximo estudio sería como las exportaciones mineras influyen en el IGBVL.

REFERENCIAS BIBLIOGRÁFICAS

Aguilar, R. \& Valdivia, D. (2011). Bolivian natural gas export prices: Modeling and forecast pooling. 
Munich Personal RePEc Archive. Recuperado de: https://mpra.ub.uni-muenchen.de/35485/

Alvarado, A., Campoverde, S. \& Merchan, P. (2014). Medición del impacto económico de la crisis financiera mundial sobre el sector petrolero exportador ecuatoriano, Revista Tecnológica ESPOL.

Al-Zyoud, H. \& Elloumi, F. (2017). Dynamics of Canadian Trade Pattern: A Time-Series Analysis, International Journal of Economics and Finance, 9(3), 115 - 125.

Amaris, G., Ávila H. \& Guerrero, T. (2017). Aplicación de modelo ARIMA para el análisis de series de volúmenes anuales en el río Magdalena, Revista Tecnura, 21(52), $88-101$.

Banco Central de Reservas del Perú- BCRP. (2016a). Memoria Anual 2015 - Balanza de Pagos. BCRP, Lima.

Banco Central de Reservas del Perú - BCRP. (2016b). Notas de Estudios del BCRP - Actividad Económica. BCRP, Lima.

Banco Mundial. (2017). PIB (US\$ a precios actuales). Grupo Banco Mundial, Washington.

Glave, M. (2007). La minería peruana: Lo que sabemos y lo que aún nos falta por saber, Group for the Analysis of Development: Investigación, políticas y desarrollo en el Perú.

Benavente, M. (2017). Efecto de la Falta de Acuerdos Arancelarios en Exportaciones de Atún Ecuatoriano a la Unión Europea: Análisis de Tendencia en el período 2010-2015, INNOVA Research Journal, 2 (5), 115 - 124.
Lanteri, L. (2010). Modelos de VAR alternativos para pronósticos (VAR Bayesianos y FAVAR): el caso de las exportaciones argentinas, Economía, 33(66) 42 - 64.

López, D. \& Martínez, C. (2013). Modeling video transmission losses using SARIMA and ARIMA models, Tecnura. 17(37).

Nolazco J., Lengua-Lafose, P. \& Céspedes, N. (2016). Contribución de los choques externos en el crecimiento económico del Perú: un modelo semi-estructural. Asociación Peruana Economía, Lima.

Seminario, B. (2016). El desarrollo de la economía peruana en la era moderna: precios, población, demanda y producción desde 1700. Universidad del Pacifico, Lima.

Tramontana, R. (2018). BVL: Es muy poco probable que mercado local sea reclasificado a frontera, Diario Gestión.

Valdés, H., Azuara, O., Corrales, M., González, A., Di Tata, J., Haarsager, U., Mendieta, M., Molina, A., Palomino, A., Ramírez, A. \& Ramírez-Goldin, A. (2016). Evaluación del programa de país: Perú 2012 - 2016. BID

World Trade Organization- WTO. (2017). World Trade Statistical Review, (WTO, Ginebra.

World Trade Organization- WTO. (2018). World Trade Outlook Indicator (WTOI), WTO, Ginebra.

Yaglom, A. (1955). Correlation theory of processes with random stationary nth increments, Matematicheskii Sbornik, 79(1), 141 - 196. 